\title{
Observation and backward trajectory of an inertio-gravity wave in the lower stratosphere
}

\author{
A. Hertzog ${ }^{1, *}$, C. Souprayen ${ }^{1}$, and A. Hauchecorne ${ }^{1}$ \\ ${ }^{1}$ Service d'aéronomie du CNRS, Verrières-le-Buisson, France \\ *Present address: Laboratoire de Météorologie Dynamique, École Polytechnique, F-91 128 Palaiseau Cedex, France
}

Received: 19 December 2000 - Revised: 20 July 2001 - Accepted: 7 August 2001

\begin{abstract}
A Doppler lidar observation of an inertio-gravity wave in the mid-latitude lower stratosphere is presented. The wave packet characteristics (vertical and horizontal wavenumbers, intrinsic and apparent frequencies) are inferred from the analysis of the hodograph of the horizontal wind fluctuations. Those parameters are used as initial conditions for the calculation of the wave packet trajectory backwards in time in the atmosphere. These calculations are realized by ray-tracing techniques, with background fields (wind and stability) provided by the European Center for Medium-Range Weather Forecasting analyses. Sensitivity tests are performed in order to estimate the robustness of the computed trajectory. It is argued that the generation of the wave has taken place in the upper troposphere, where evidence of large synoptic scale Rossby wave disturbances are found. Our results support the fact that geostrophic adjustment (and possibly shear instabilities) associated with such disturbances could be an effective mechanism for the generation of inertia-gravity waves in the mid-latitude.
\end{abstract}

Key words. Meteorology and atmospherid dynamics, mesoscale meteorology, waves and tides, instruments and techniques

\section{Introduction}

In the atmosphere, gravity waves are thought to be primarily generated in the troposphere. Due to the exponential decrease in density with altitude, their upward propagation is associated with an increase in their amplitude. In the upper stratosphere and mesosphere, criteria of static or dynamic instability may be reached so that the waves break and deposit their momentum and energy in the background flow. This force (which generally acts as a drag) is crucial in maintaining the mean thermodynamic structure of the middle atmosphere: it is considered to be the driver of the merid-

Correspondence to: A. Herzog

(hertzog@lmd.polytechnique.fr) ional Brewer-Dobson circulation in the middle atmosphere through the "downward control" principle, leading to an upwelling in the summer pole and a downwelling in the vortex of the winter pole (e.g. Holton et al., 1995).

However, recent studies have underlined the role played by gravity waves in the stratosphere. First, Alexander and Rosenlof (1996) stated that general circulation models (GCM) lack an accelerating force to accurately represent the stratospheric jet. This force may result from the breaking of non-stationary gravity waves. In addition, the general concern about ozone depletion in the mid-latitudes has raised the question of the polar vortex permeability, which is generally attributed to the interaction between Rossby waves and the background flow (e.g. Waugh et al., 1994; Bowman, 1996). Nevertheless, inertia-gravity waves, whose frequency is low, may induce significant horizontal displacements and participate in the transport of ozone depleted air through the vortex edge. For instance, Danielsen et al. (1991) noted the presence of fluctuations in ozone profiles near the vortex edge, which they attributed to the effects of an inertio-gravity wave. This transport is, however, irreversible only if the wave experiences some breaking. Finally, vertical and horizontal displacements, induced by gravity waves, may also contribute to the dispersion of tracers in the mid-latitude stratosphere (the so-called "surf zone") (e.g. Teitelbaum et al., 1994, 1996; Gibson-Wilde et al., 1997; Eckermann et al., 1998).

Several mechanisms susceptible of generating gravity waves have been identified. The most common one is certainly the emission of waves by a flow blowing over orography (e.g. Nastrom and Fritts, 1992; Lott and Teitelbaum, 1993). Shear instability (Lalas and Einaudi, 1976; Fritts, 1982; Fritts and Nastrom, 1992; Sutherland and Peltier, 1995; Lott, 1997), convection (Lu et al., 1984; Pfister et al., 1986), frontal acceleration and geostrophic adjustment (Blumen, 1972; Ley and Peltier, 1978; Van Tuyl and Young, 1982; Gall et al., 1988; Koch and Dorian, 1988; Fritts and Nastrom, 1992; Fritts and Luo, 1992; Luo and Fritts, 1993; Eckermann and Vincent, 1993; O'Sullivan and Dunkerton, 1995; Griffiths and Reeder, 1996; Reeder and Griffiths, 1996; 
Thomas et al., 1999; Ford et al., 2000) have also been invoked. The relative importance of each mechanism certainly depends on not only the geographical location (e.g. emission by convective activity in the equatorial region or lee waves above orography), but probably also the time of the year. Moreover, each process generates waves with specific characteristics (e.g. stationary waves are emitted above orography, if the mean wind is steady). Therefore, a precise knowledge of the gravity-wave field and its possible variations is needed to build accurate parameterizations of its effect on global-scale dynamics inside general circulation models (GCM).

In this study, we report on an observation realized by a Doppler lidar of an inertio-gravity wave in the lower stratosphere. It is suggested in our measurements, as well as in other published studies, that inertia-gravity waves are a major component of the wave field in the mid-latitude lower stratosphere (e.g. Thompson, 1978; Cadet and Teitelbaum, 1979; Sato and Woodman, 1982; Sato, 1989; Thomas et al., 1992; Sato, 1994; Hertzog et al., 2001). Our goal here is to gain insight into the process that may be responsible for the presence of the observed wave. For this purpose, we have developed a 3-D, non-stationary ray-tracing model for gravity waves. The model is used to compute the backward trajectory of the wave packet in the lower stratosphere and troposphere. Such calculations enable us to identify the region of the flow where the packet is presumed to be generated.

The rest of the paper is divided as follows: in Sect. 2, the Doppler lidar is briefly described and the observation of the inertio-gravity wave packet is presented. In Sect. 3, the raytracing equations for gravity waves are recalled and some insights into the implementation of the model are given. In Sect. 4, the back-trajectory of the wave packet is computed and some sensitivity tests are performed in order to check the robustness of the calculations. Section 5 is devoted to a discussion concerning the generation processes which may have taken place along the wave trajectory. Finally, in Sect. 6, we summarize our main findings and give some concluding remarks.

\section{Observation}

\subsection{Instrument and data set}

The measurements presented in this study were made with an incoherent Rayleigh-Mie Doppler lidar, located at the Observatory of Haute-Provence (OHP), in the south of France $\left(44^{\circ} \mathrm{N}, 6^{\circ} \mathrm{E}\right)$. A complete description of the instrument, some validation tests and first climatological results can be found in Souprayen et al. (1999). The lidar is able to measure both components of the horizontal wind velocity from the mid-troposphere to the stratopause. The measurement accuracy depends on the duration of temporal integration and vertical resolution. A typical error bar of $2-3 \mathrm{~m} \mathrm{~s}^{-1}$ can be achieved in the lower stratosphere with a vertical resolution of $230 \mathrm{~m}$ and an integration time of $15 \mathrm{~min}$ (Hertzog et al., 2001).

The lidar is operated approximately twice a week and measurements are limited to nighttime and clear sky conditions. This paper focuses on the night of 3 September 1997. The mean profile obtained in the stratosphere between 3 September at 20:00 UTC and 3 September at 04:00 UTC is shown in Fig. 1 that time, the tropospheric data, which come from independent optical channels, were erroneous and, therefore, are not shown.

Also shown in Fig. 1 are high-resolution, radiosounding profiles at 23:00 UTC on 3 September 1997 in Nîmes (100 km westward of the observatory), the European Center for Medium-range Weather Forecast (ECMWF) T106 model analysis, and a 6-hour forecast interpolated at the observatory at 18:00 UTC on 3 September 1997 at 00:00 UTC and 06:00 UTC on 4 September 1997.

The agreement between the sonde and the lidar is very good, although the sonde profile presents more structures. However, it must be noted that the lidar profile results from an 8-hour integration, which can smooth high-frequency fluctuations. The overall best agreement between the lidar and the model is found with the 00:00 UTC analysis: the two data sets are very close for the meridional wind, but the analyzed zonal wind is $\sim 5 \mathrm{~m} \mathrm{~s}^{-1}$ weaker than the measured one around $15 \mathrm{~km}$. Finally, the tropospheric jet stream is southward and is quite weak $\left(\sim 15 \mathrm{~m} \mathrm{~s}^{-1}\right)$.

Around $18-20 \mathrm{~km}$, the radiosonde detected significant fluctuations in both components of the wind velocity $\left(\sim 5 \mathrm{~s}^{-1}\right)$. In order to capture those fluctuations, the lidar data were re-treated with a $15 \mathrm{~min}$ integration time. Each of the 15 min profiles were then filtered between 1.3 and $5 \mathrm{~km}$ in order to remove the mean structure of the wind (long wavelengths) and the measurement noise (short wavelengths). The resulting time-altitude plot is shown in Fig. 2.

A clear oscillation with a maximum amplitude of $\sim 6 \mathrm{~m} \mathrm{~s}^{-1}$ located near $20 \mathrm{~km}$ with descending phase can be seen in both components of the horizontal wind. Such features are generally attributed to propagating inertia-gravity waves (e.g. Thomas et al., 1999; Hertzog et al., 2001).

The apparent period of the velocity oscillations (i.e. the period measured by an instrument that is fixed relative to the ground), which is $\approx 16 \mathrm{~h}$, is twice as long as the measurement duration, which explains why the wave was hardly visible on the mean lidar profiles. The vertical wavelength is $\sim 2 \mathrm{~km}$, although in the lower part of the packet, the wavelength seems to be shorter. Moreover, it seems that the oscillations are disappearing below $18 \mathrm{~km}$ at the end of the measurement, which can be attributed to the upward propagation of the wave packet. Indeed, since the mean wind is weak at that altitude, the Doppler shift between the apparent and intrinsic frequencies may also be weak, so that upward propagating gravity waves may effectively present a descending phase relative to the ground. Such low-frequency, lowwavenumber waves are frequently observed by the Doppler lidar in the lower stratosphere. Observations previously made by radar or balloons have also underlined their ubiq- 
(a)

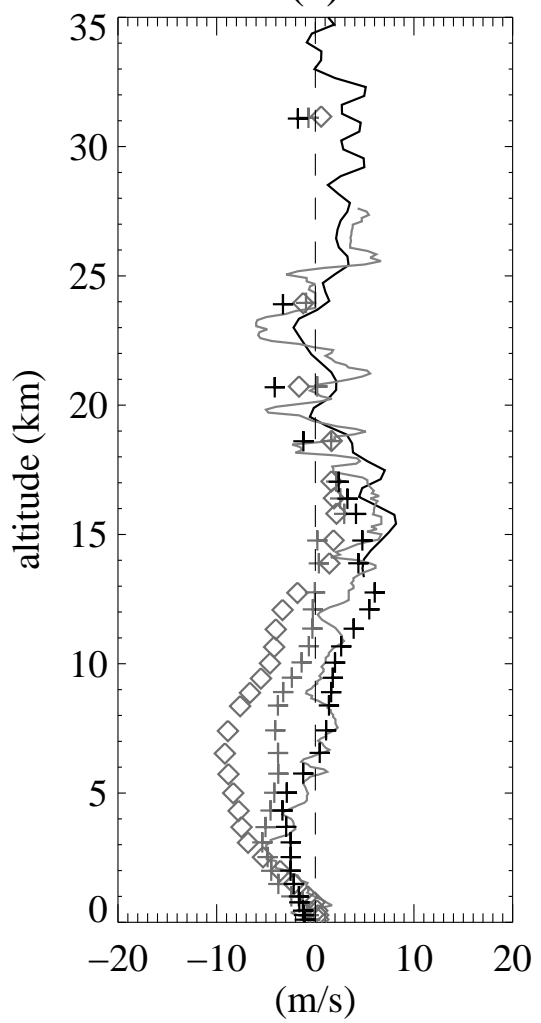

(b)

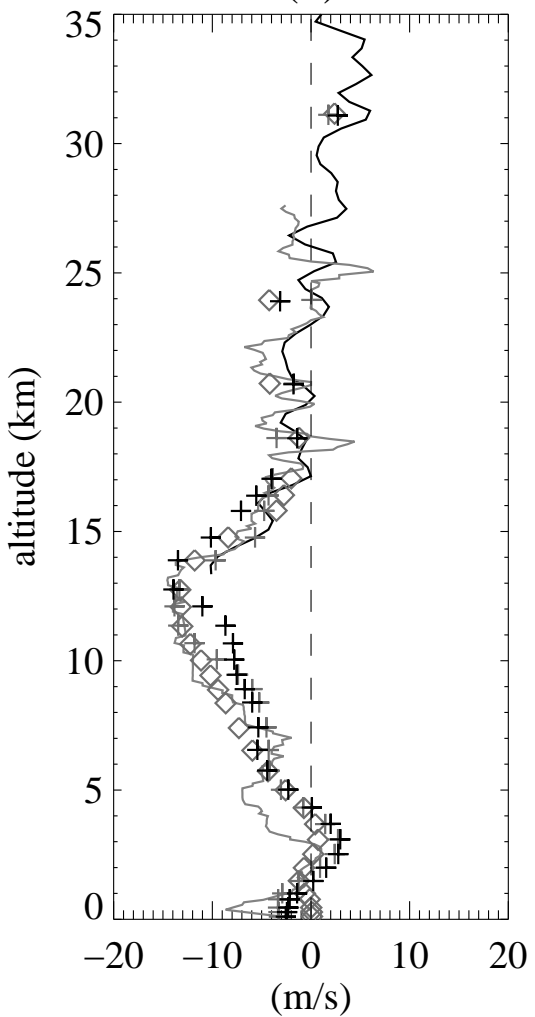

Fig. 1. Zonal ((a), positive eastward) and meridional ((b), positive northward) wind measured by the Doppler lidar (solid black) on 3 September 1997 (20:00 UTC-04:00 UTC) and by the radiosonde (solid grey) at Nîmes at 23:00 UTC. Also shown are the ECMWF profiles interpolated at the OHP (18:00 UTC on 3 September 1997 (diamond), and 00:00 UTC (grey crosses) and 06:00 UTC (black crosses) on 4 September 1997).

(a)

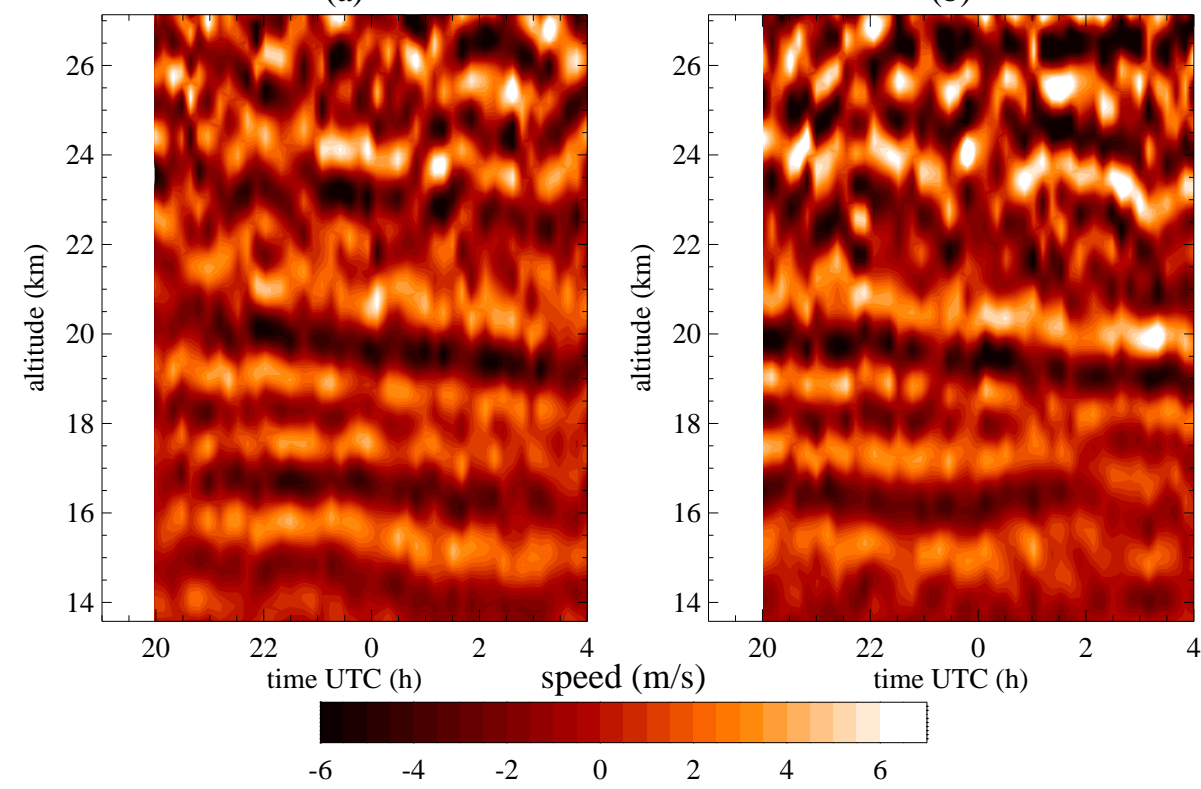

Fig. 2. Time-altitude plot of the zonal (a) and meridional (b) components of the horizontal-wind velocity measured by the Doppler lidar at OHP on 3 September 1997. 


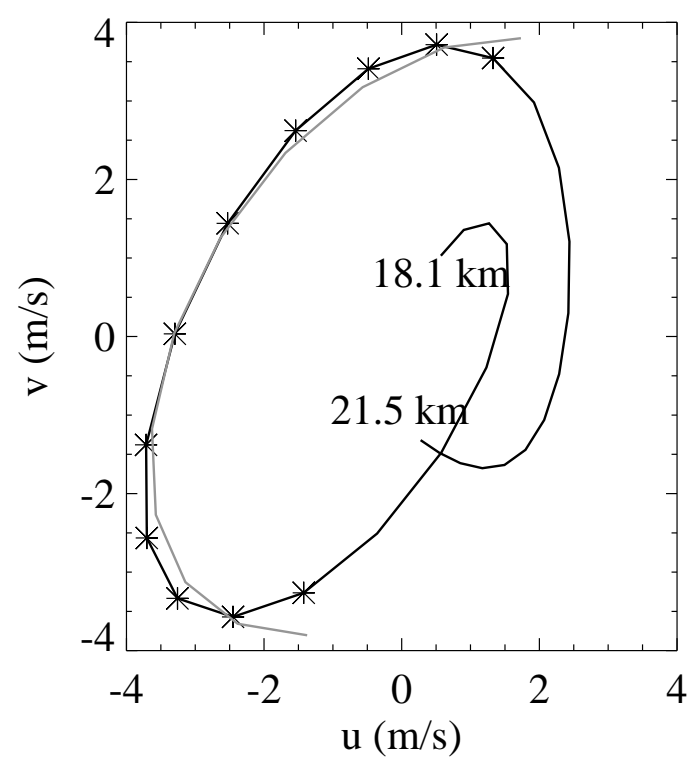

Fig. 3. Hodograph (black) of the wind fluctuations generated by the gravity wave. The stars show the region where the fit was made and the grey curve is the result of the fit (see text).

uitous presence in this part of the atmosphere (e.g. Thompson, 1978; Cadet and Teitelbaum, 1979; Sato and Woodman, 1982; Sato, 1989; Thomas et al., 1992; Sato, 1994).

\subsection{Hodograph and characteristics of the wave}

The main characteristics of the observed wave packet (i.e. intrinsic frequency, vertical wavenumber, direction of propagation) were estimated through the analysis of its hodograph. A temporal integration of $4 \mathrm{~h}$, centered at 02:00 UTC was performed in order to reduce the noise level without removing the fluctuations due to the wave. The same filter was applied as in the previous section and the resulting hodograph which is between $18.1 \mathrm{~km}$ and $21.5 \mathrm{~km}$ is shown in Fig. 3. The hodograph is obviously elliptic and the wind vector rotates clockwise with altitude, which is consistent with the hypothesis of an upward-propagating gravity wave in the northern hemisphere.

According to the polarization relationships, the zonal $\left(u^{\prime}\right)$ and meridional $\left(v^{\prime}\right)$ components of the wind velocity fluctuations induced by inertia-gravity waves may be written as:

$\left[\begin{array}{l}u^{\prime} \\ v^{\prime}\end{array}\right]=\hat{u}[R(\Theta)]\left[\begin{array}{c}\cos (m z+\Phi) \\ -\frac{f}{\omega_{0}} \sin (m z+\Phi)\end{array}\right]$

where $\hat{u}$ is the amplitude of the wave, $m$ and $\omega_{0}$ are its vertical wavenumber and intrinsic frequency (frequency measured by an observer moving with the wind), $f$ is the Coriolis parameter (Andrews et al., 1987). $[R(\Theta)]$ is the rotation which allows for a reference frame where the phase speed of the wave is parallel to the $x$-axis. Equation (1) states that the ratio between the long and short ellipse axes is equal to $\omega_{0} / f$. The hodograph was fitted with the help of Eq. (1) between $19.0 \mathrm{~km}$ and $20.2 \mathrm{~km}$, where the envelope of the wave
Table 1. Gravity wave packet characteristics

\begin{tabular}{cccccc}
\hline$k\left(\mathrm{~m}^{-1}\right)$ & $l\left(\mathrm{~m}^{-1}\right)$ & $m\left(\mathrm{~m}^{-1}\right)$ & $\omega_{0} / f$ & $\omega / f$ & $\Theta$ \\
\hline $1.110^{-5}$ & $1.210^{-5}$ & $-2.810^{-3}$ & 1.6 & $\sim 1.0$ & $48^{\circ}$ \\
\hline
\end{tabular}

packet can be considered as approximately constant. Thus, the fit gives an estimate of the vertical wavelength, the direction of propagation and the intrinsic frequency of the wave.

The result of this fit is shown in Table 1. The zonal $(k)$ and meridional $(l)$ wavenumbers were estimated from the dispersion relationship:

$m^{2}=\frac{N^{2}-\omega_{0}^{2}}{\omega_{0}^{2}-f^{2}}\left(k^{2}+l^{2}\right)$

with $N^{2}$ as the Brunt-Väisälä frequency and knowing that:

$|\tan \Theta|=\left|\frac{l}{k}\right|$.

Equation 2 is valid as long as the vertical wavelength of the wave is small compared to twice the density scale height (i.e. $m \gg 1 / 2 H)$, which is true for the case that we study. Otherwise, the dispersion relationship includes non-hydrostatic waves.

However, an ambiguity of $180^{\circ}$ remains for the direction of the horizontal wave vector. This ambiguity can be resolved if there exists a Doppler shift between the wave intrinsic and apparent frequencies $(\omega)$, both of which are linked through:

$\omega=\omega_{0}+\boldsymbol{k}_{\boldsymbol{h}} \cdot \boldsymbol{u}$

with $\boldsymbol{u}$ as the mean wind vector and $\boldsymbol{k}_{\boldsymbol{h}}=(k, l)$ as the horizontal wave vector. Now, the intrinsic frequency is given by the hodograph analysis (see Eq. 1), whereas the apparent frequency may be estimated from the observations (e.g. Fig. 2). Thus, when $\boldsymbol{k}_{\boldsymbol{h}} \cdot \boldsymbol{u} \neq 0$, the direction of $k_{h}$ can be determined with the help of Eq. 4. At $19.5 \mathrm{~km}$, the zonal wind is almost vanishing, while the meridional component is weakly negative. Thus, since the hodograph analysis gives an estimate of the intrinsic frequency of $\sim 1.6 f$, whereas the observations tend to show that $\omega \sim f$, it follows that $l>0$. Furthermore, since the wave vector is aligned with the long axis of the hodograph, $l>0$ implies that $k>0$, so that the horizontal wave vector is directed toward northeast.

\section{Ray-tracing model}

\subsection{Equations and numerical aspects}

The ray-tracing model for gravity waves is used for computing the trajectory of the observed wave packet back to the region of the flow where it was emitted. The ray-tracing equations are (Jones, 1969; Lighthill, 1978):

$$
\frac{d x_{i}}{d t}=\frac{\partial \omega}{\partial k_{i}} \quad(i=1,2,3)
$$


(a) altitude

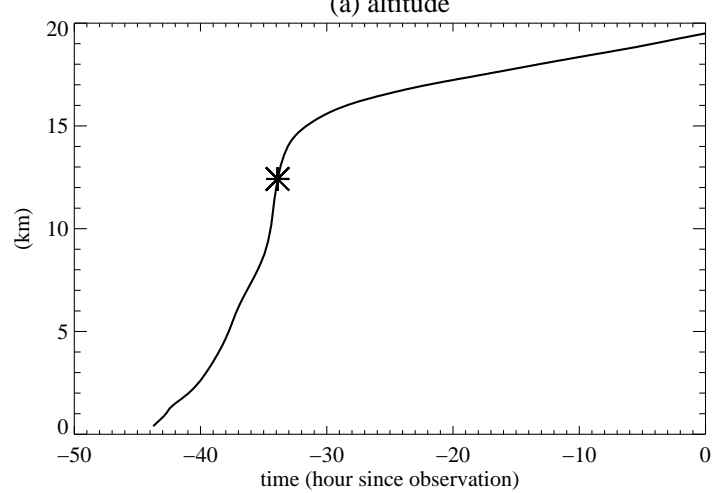

(c) zonal and meridional wavelengths

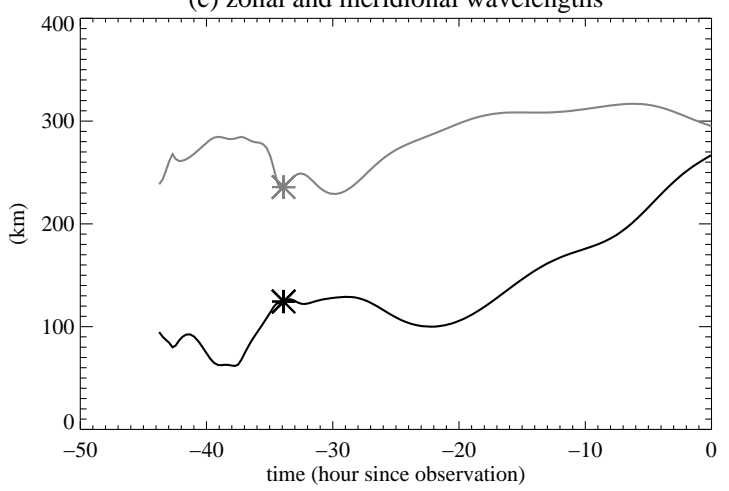

(e) action density

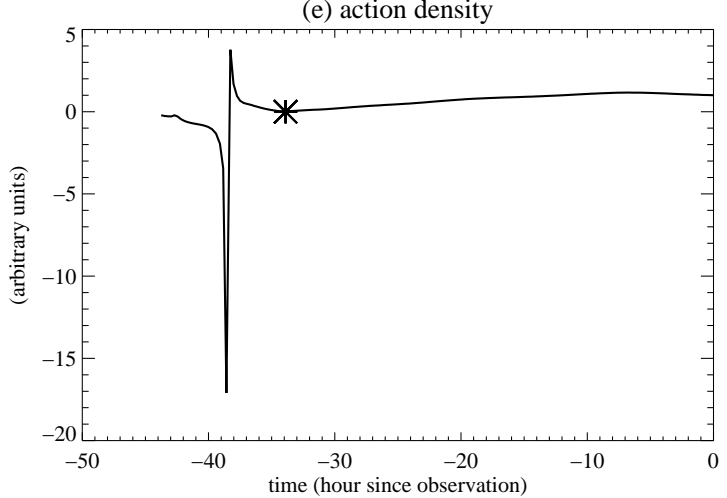

(g) Richardson number

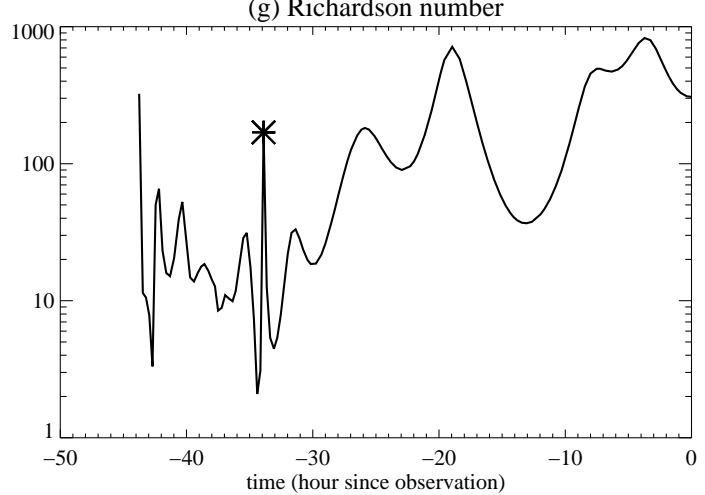

(b) vertical wavelength

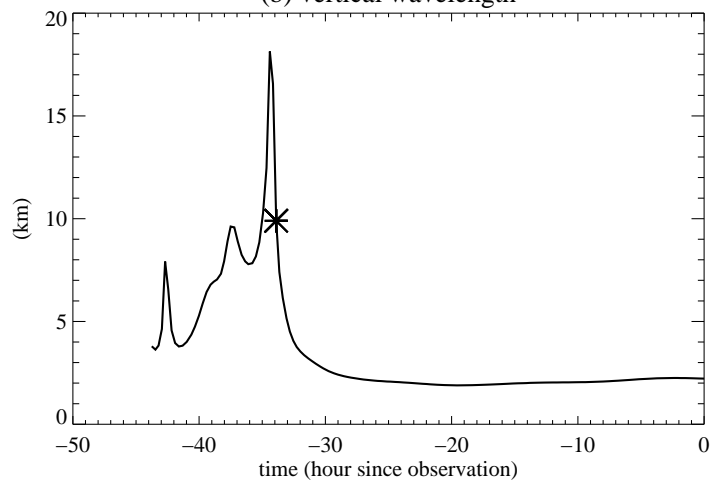

(d) intrinsic and apparent frequencies
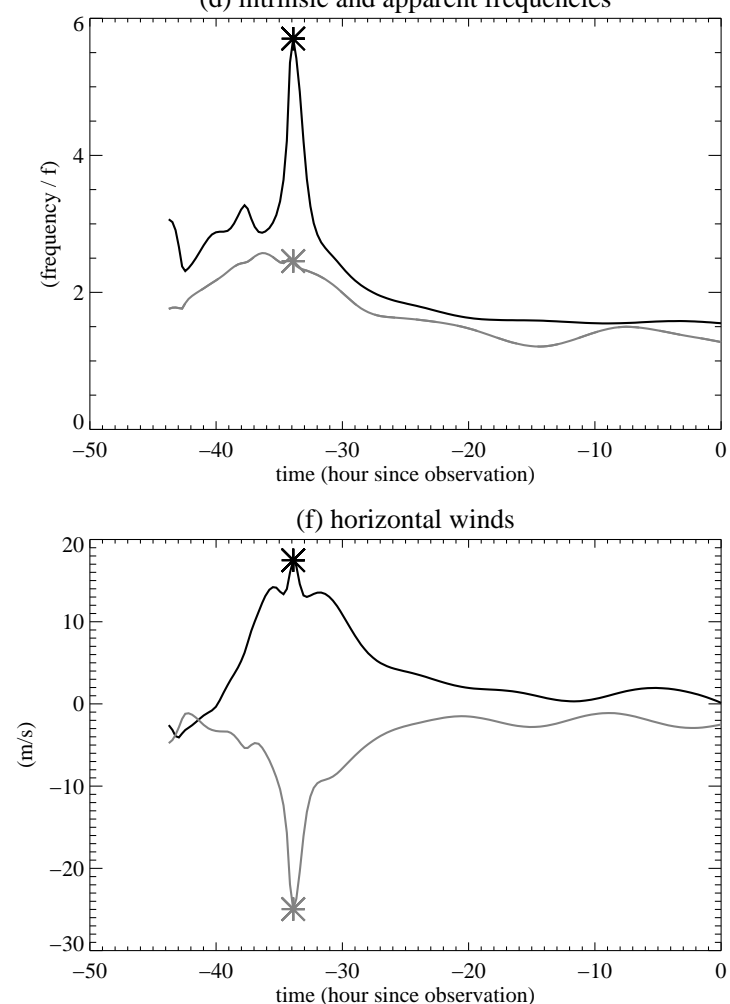

(h) WKB parameters

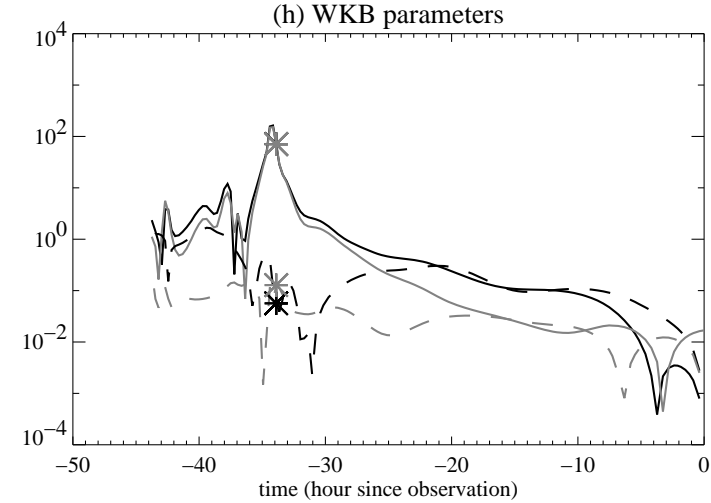

Fig. 4. Results of the backward ray-tracing simulation: (a) wave packet altitude, (b) vertical, (c) zonal (black) and meridional (grey) wavelengths, (d) intrinsic (black) and apparent frequency (grey), (e) action density; (f) zonal (black, positive eastward) and meridional (grey, positive northward) wind velocities, (g) Richardson number and (h) $\alpha$ (blacked dashed), $\beta$ (grey dashed), $\gamma$ (black) and $\delta$ (grey) (h) along the ray. The stars show the supposed time of generation (see text). 


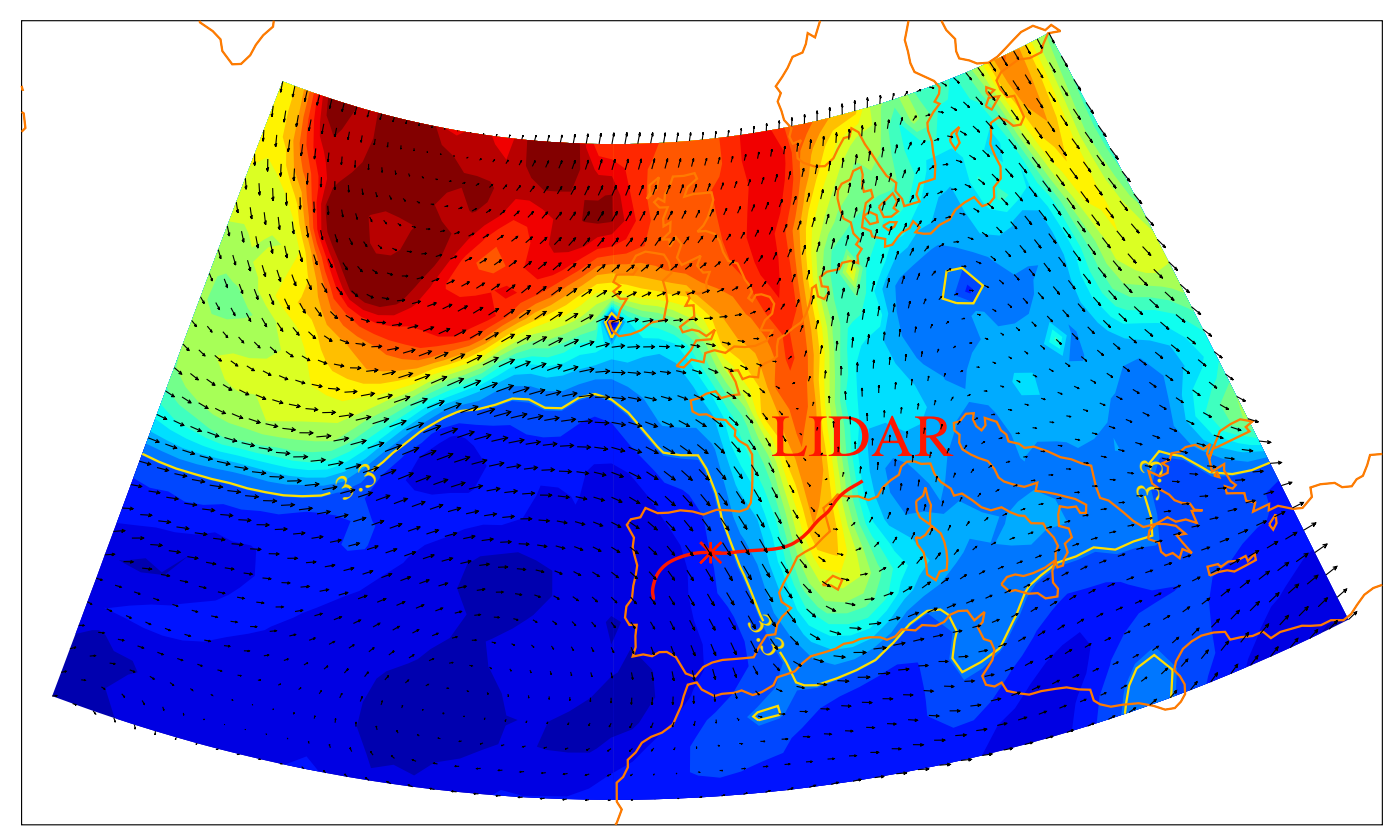

Fig. 5. Horizontal projection of the gravity wave trajectory (red). The starting point of the backward ray-tracing calculations is referred to as "LIDAR". The contours represents the MPV field on the $340 \mathrm{~K}$ isentrope at the time when the observed ray crossed it (2 September 1997 at 14:00 UTC). The star shows the ray intersection with that isentrope. Also shown is the horizontal wind field on the same surface. The indicated MPV contour corresponds to a PV value of 2 PV units (MPV = 3.3 PV unit), that approximately indicates the tropopause. High(low-) PV values are color-coded in red (blue).

$\frac{d k_{i}}{d t}=-\frac{\partial \omega}{\partial x_{i}}$

with $\boldsymbol{x}=\left(x_{1}, x_{2}, x_{3}\right)$ as the wave packet position, and $(k, l, m)=\left(k_{1}, k_{2}, k_{3}\right)$ as the zonal, meridional and vertical wavenumbers. This set of equations is complemented by the Doppler shift Eq. (4) and the dispersion Eq. (2). System (5) is developed in Appendix A in a 3-D, non-stationary framework. The first equation of the system states that the wave packet trajectory is given by its ground-based group velocity, while the second equation expresses the refraction of the wave vector along the trajectory due to the inhomogeneities of the propagating medium (wind shear, variations of $N^{2}$ ).

The ray-tracing equations are integrated by a fourth order Runge-Kutta scheme with adaptive time steps (Press et al., 1989). Background fields (horizontal wind and static stability) necessary for the integration are given by the ECMWF analyses. The ECMWF model version used in this study has a T106 spectral truncation (equivalent horizontal resolution of $1.125^{\circ} \times 1.125^{\circ}$ ) and 31 levels in the vertical, ranging from the ground to $10 \mathrm{hPa}$. Analyses and short-term forecasts are available every 6 hours. The fields are then interpolated at the wave packet position $(\boldsymbol{x}, t)$ using cubic splines.

\subsection{Control of integration validity}

First, the accuracy of the numerical scheme presented above is checked. A convenient means for doing this is to compare the wave packet frequency evolution obtained from the dispersion relationship and the direct estimation of $\omega$, which may be computed through the integration of:

$$
\frac{d \omega}{d t}=\frac{\partial \omega_{0}}{\partial N^{2}} \frac{\partial N^{2}}{\partial t}+\boldsymbol{k} \cdot \frac{\partial \boldsymbol{u}}{\partial t}
$$

in the same way as Eq. (5). In the simulations presented below, both estimations do not differ more than $1 \%$ and, therefore, give some confidence in the numerical scheme.

Second, we must also check that the WKB approximation remains valid during the integration. This approximation states that the atmospheric variation must occur on scales larger than the characteristic scale of the wave. The WKB approximation is necessary for the derivation of system (5). As in Marks and Eckermann (1995) and Eckermann and Marks (1996), the validity of this assumption is checked by computing the following parameters:

$$
\begin{gathered}
\alpha=\frac{1}{k^{2}}\left|\frac{\partial k}{\partial x}\right| \\
\beta=\frac{1}{l^{2}}\left|\frac{\partial l}{\partial y}\right| \\
\gamma=\frac{1}{m^{2}}\left|\frac{\partial m}{\partial z}\right| \\
\delta=\frac{1}{\omega_{0}^{2}}\left|\frac{\partial \omega_{0}}{\partial t}\right|
\end{gathered}
$$

which, respectively, measure the local rate of variation of the zonal, meridional, vertical wavenumbers and intrinsic frequency. Those parameters are, therefore, computed during the integration of the ray equations and enable us to control 
the validity of our results (see Fig. 4 and the discussion in the following section). Finally, the action density of the wave packet is also computed, according to equations presented in Appendix A.

\section{Results of the ray simulation}

\subsection{Observed wave trajectory and characteristics}

At 02:00 UTC on 3 September $1997\left(t_{0}\right)$ and at an altitude of $19.5 \mathrm{~km}\left(z_{0}\right)$, a wave packet, whose initial characteristics are defined in Table 1, was launched. For simplicity, this simulation will be referred to as the "observed ray" in the following discussion. The integration of the ray-tracing equations was made backwards in time in order to investigate the wavegeneration processes. The results are described in Fig. 4 and the horizontal projection of the ray is shown in Fig. 5.

The calculations were stopped when the wave reached the ground. For the first 30 hours of integration, the wave propagated from the southwest without a significant change in its characteristics. However, when it encountered the tropospheric jet stream at around $15 \mathrm{~km}$, the vertical group velocity suddenly increased as well as the vertical wavelength and the intrinsic frequency. The jet stream presents at the displayed time an important southward component. Then the Richardson number became relatively small (i.e. 2) while the wave was propagating in the shear layer associated with the tropospheric part of the jet stream. In the troposphere, the wave trajectory curved southward and reached the ground above Portugal. At the ground, the wind was directed toward the southwest.

It should also be noted that the action density changed its sign $\approx 38 \mathrm{~h}$ before the observation time, which meant that the wave encountered a caustic at that time (Broutman, 1986). This is further confirmed by the $\gamma$ and $\delta$ parameters which become larger than 1 , in agreement with the sudden variations in the vertical wavenumber and intrinsic frequency. At the caustic, the WKB assumption is no longer valid and the action density singularity is a failure of the raytracing equations. Nevertheless, Broutman (1986) shows that the ray-tracing equations can still produce valid results after the caustic.

At the caustic, however, the energy of the wave packet is concentrated in a small, physical space, so that the amplitude of the induced fluctuations increases and instabilities may occur. If they have enough time to develop, the instabilities may totally destroy the wave packet, thus inhibiting any propagation. We shall see in the next section that the emission of the wave packet may nonetheless have taken place before (above) the caustic.

Figure 5 also shows the reconstructed modified potential vorticity (MPV) field on the $340 \mathrm{~K}$ isentrope at the time when the observed ray crossed this surface (i.e. 2 September 1997, 14:00 UTC). The MPV is simply related to the potential vor-

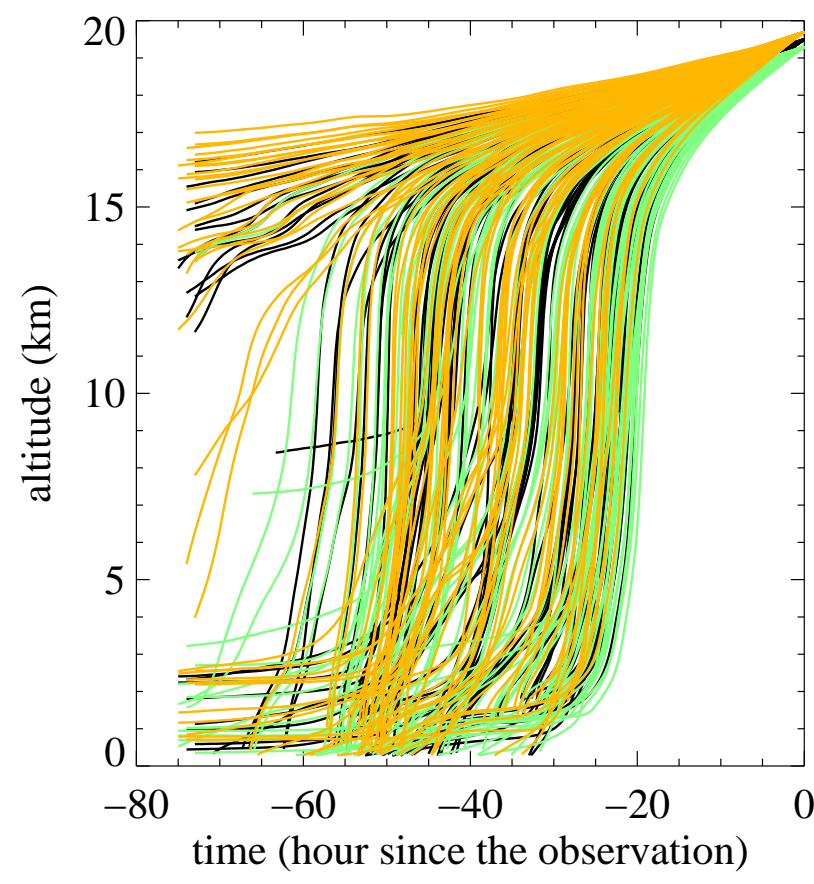

Fig. 6. Time-altitude plot of the 243 rays for which the initial conditions have been modified (green: rays which start at $19.3 \mathrm{~km}$, black: $19.5 \mathrm{~km}$, orange: $19.7 \mathrm{~km}$.)

ticity (PV) by:

$M P V=P V\left(\frac{\theta}{\theta_{0}}\right)^{-4.5}$

with $\theta$ as the potential temperature and $\theta_{0}=380 \mathrm{~K}$ (Lait, 1994). The MPV is designed to suppress the exponential increase in PV with altitude. The intersection between the ray and the $340 \mathrm{~K}$ isentrope is shown with a star on this figure. This intersection lies near the main gradient of MPV, which separates the troposphere (low-MPV values) from the stratosphere (high-MPV values) (e.g. Holton et al., 1995). It appears on Fig. 5 that the tropopause is very disturbed above western Europe on 2 September 1997: a large streamer of high-MPV values is extending southward into the troposphere, far from the main stratospheric reservoir. Associated with this trough, a significant disturbance of the jet stream occurred: a jet streak had formed southwest of the Britannic islands, which corresponds to the maximum MPV gradient. The flow is then deflected southward, in the so-called "jet exit" region. On the eastern flank of the ridge, the flow becomes northward and the wind speed is weaker than on the western flank. Such synoptic structure of the tropopause results from baroclinic activity in the upper troposphere (e.g. Thorncroft et al., 1993).

\subsection{Robustness of the integration}

We have already noted that both methods for computing the wave packet frequency give exactly the same results, indi- 


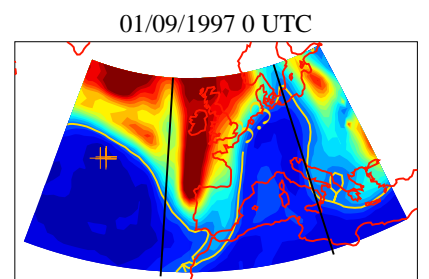

01/09/1997 18 UTC

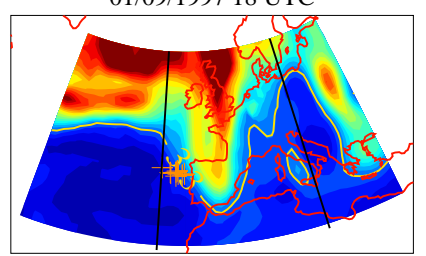

02/09/1997 12 UTC

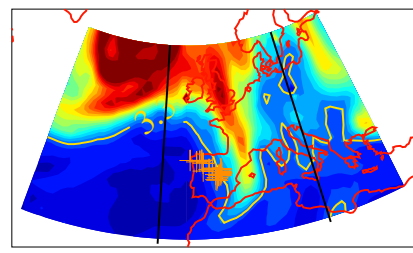

03/09/1997 6 UTC

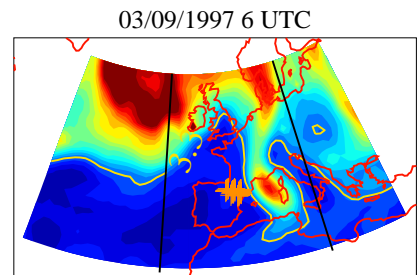

04/09/1997 0 UTC

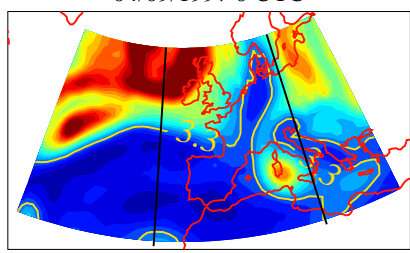

01/09/1997 6 UTC

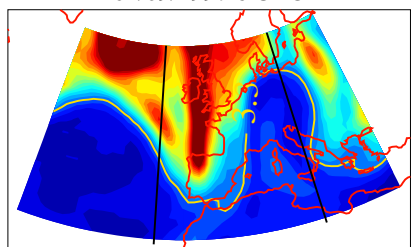

02/09/1997 0 UTC

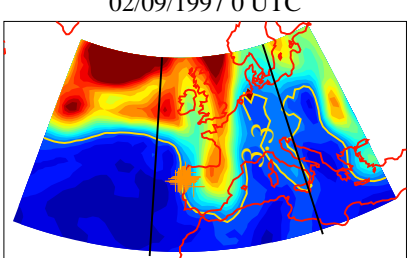

02/09/1997 18 UTC

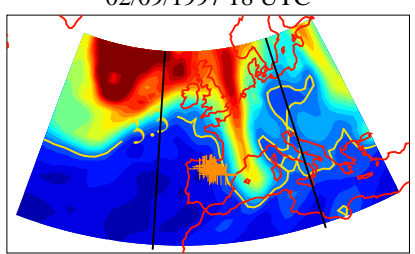

03/09/1997 12 UTC

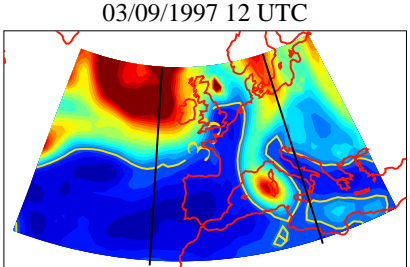

04/09/1997 6 UTC

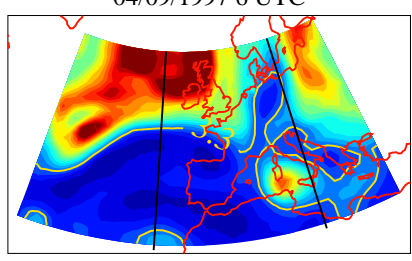

01/09/1997 12 UTC

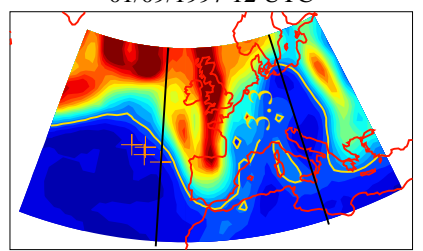

02/09/1997 6 UTC

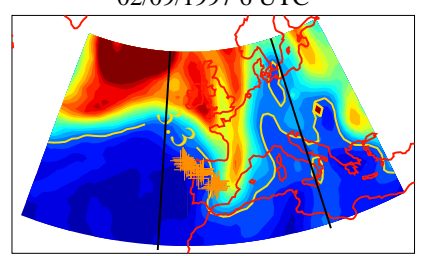

03/09/1997 0 UTC

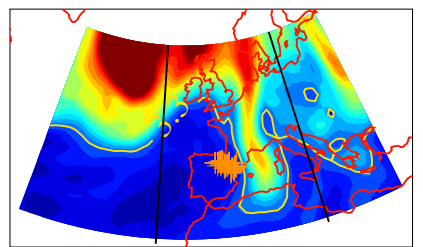

03/09/1997 18 UTC
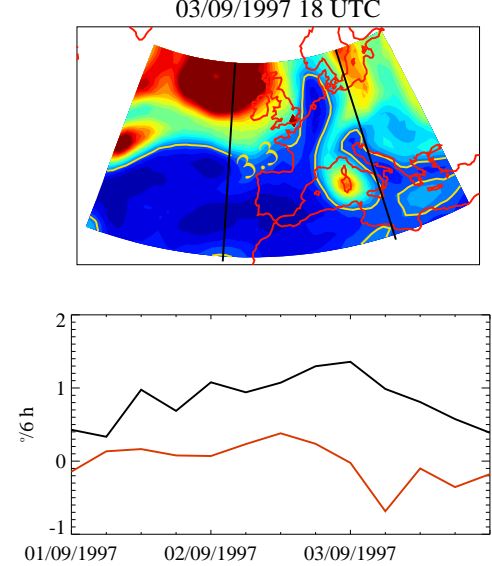

Fig. 7. Evolution of the MPV field on the $340 \mathrm{~K}$ isentropic surface. The indicated MPV contour is the same as in Fig. 5. Rays that intersect the $340 \mathrm{~K}$ isentrope at a time roughly corresponding to the analysis time are shown by crosses. Limits for the calculation of the field crosscorrelation are indicated by thick solid lines at $-15^{\circ} \mathrm{E}$ and $15^{\circ} \mathrm{E}$. The last picture represents the displacement speed of the MPV field on the isentrope (black: eastward velocity, red: northward velocity).

cating that the integration scheme is accurate and, therefore, may not be the major source of integration error.

Another possible source of error may be induced by the ECMWF background fields. In particular, when the meteorological situation is very disturbed, as it is the case here at the tropopause, the model resolution (either horizontal, vertical or temporal) might be too weak to resolve the ageostrophic circulation which takes place. However, the event we investigated took place in northern Europe, where many observations are available and where the ECMWF model is the most trustful. We may indeed assume that for analysis or short term prediction, the bias between modeled and real winds does not exceed $\sim 1.5 \mathrm{~m} \mathrm{~s}^{-1}$, regardless of the altitude (Andersson et al., 1998). Locally, however, especially close to the jet core, the difference may be larger. Given an integra- tion time of $\sim 40 \mathrm{~h}$, the error made on the position of the wave packet is then $\sim 200 \mathrm{~km}$. We will assume that this value is also valid for the error due to the static stability field.

It is generally admitted that in ray-tracing calculations, the major source of error comes from the initial conditions, i.e. the initial wave characteristics and the integration starting point. To investigate such errors, we made sensitivity tests on several parameters (i.e. $z_{0}, t_{0}, \lambda_{z}, \omega_{0}, \Theta$ ). The only parameters which we assume to be perfectly known are the OHP latitude and longitude. The uncertainties associated with each of the five preceding parameters are presented in Table 2 and $3^{5}$ integration were performed, corresponding to all possible combinations $\left(z_{0}+\epsilon_{1} \Delta z, t_{0}+\epsilon_{2} \Delta t, \ldots\right.$ with $\left.\epsilon_{i}=-1,0,1\right)$.

The time-altitude and longitude-altitude plots of the 243 rays are presented on Fig. 6. A somewhat large dispersion 
Table 2. Assumed uncertainties on the wave-packet characteristics

\begin{tabular}{ccccc}
\hline$\Delta z$ & $\Delta t$ & $\Delta \lambda_{z}$ & $\Delta\left(\omega_{0} / f\right)$ & $\Delta \Theta$ \\
\hline $0.2 \mathrm{~km}$ & $2 \mathrm{~h}$ & $.2 \mathrm{~km}$ & .2 & $10^{\circ}$ \\
\hline
\end{tabular}

of the rays may be noted. Indeed, although 209 rays ( $86 \%$ ) penetrated into the troposphere with a path similar to that of the observed ray, the time needed for the packets to reach the $10 \mathrm{~km}$ surface varies between 20 and 60 hours. However, even if at first sight, this interval looks relatively large, therefore, casting some doubt on the significance of the raytracing calculations, we shall see in the following that the different rays actually cross the tropopause in approximately the same region of the flow. To show this, we examined the MPV evolution on the $340 \mathrm{~K}$ isentropic surface from 1 September 1997 at 00:00 UTC to 4 September 1997 at 06:00 UTC, which is shown in Fig. 7. The trough is moving westward with time and becomes more and more stretched. On 3 September 1997, the stretching has become so large that a cutoff cyclone has been created over the Mediterranean Sea.

Let $t_{a}$ be the time of an ECMWF analysis. The location where the rays that have intersected the $340 \mathrm{~K}$ isentrope in $\left(t_{a}-3 h ; t_{a}+3 h\right)$ are shown with stars on the corresponding sub-panel. It can be seen that the crosses stay relatively steady with respect to the MPV ridge: almost all crosses are located on the southwestern flank of the trough. This can be further confirmed if one considers the displacement velocity of the trough, which was obtained by computing the latitude and longitude lags that give the best MPV cross-correlation between the different analyses within the area limited by $\left(30^{\circ} \mathrm{N} ; 60^{\circ} \mathrm{N}\right)$ and $\left(-15^{\circ} \mathrm{E} ; 15^{\circ} \mathrm{E}\right)$. The result is shown on the last picture of Fig. 7: a positive, eastward velocity of $\sim 1^{\circ} 6 \mathrm{~h}$ is found, while the meridional velocity stays weak (similar displacement velocities were obtained when considering the cross-correlations of the wind meridional component). The points at which the 243 rays crossed the $340 \mathrm{~K}$ surface were then advected with this velocity backward or forward in time until the date when the observed ray crossed that isentrope. The result is shown in Fig. 8 .

It can be seen that at the synoptic scale, most of the rays penetrated in the upper-troposphere in the same region of the flow, i.e. on the southwest part of the streamer. Therefore, we believe that the observed ray is representative of the actual trajectory of the wave packet (at least until the tropopause) and that the discussion which follows does not depend critically on the initial conditions of the ray-tracing simulations.

\section{Discussion}

The issue that will be addressed here is whether it is possible to obtain some insight into the processes that led to the generation of the wave observed by the lidar at OHP. For this,

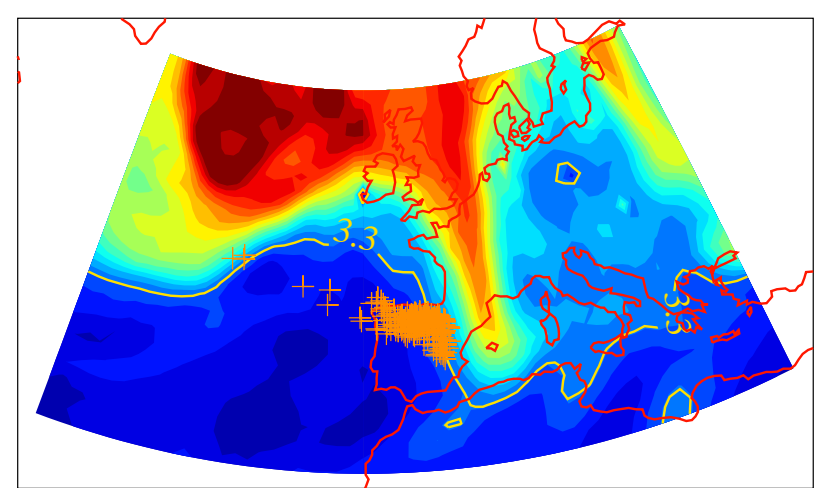

Fig. 8. MPV on the $340 \mathrm{~K}$ isentrope on 2 September 1997 at 14:00 UTC. Crosses indicate the location where the rays crossed that isentrope, after advection until this time with the displacement speed of the flow in the upper-troposphere lower-stratosphere (see text).

we will rely on the results of the ray-tracing simulation and the ECMWF analyses.

\subsection{Geostrophic adjustment}

Blumen (1972), Fritts and Nastrom (1992) and Luo and Fritts (1993) have shown that the return of a disturbed jet to geostrophic equilibrium is achieved through the emission of inertia-gravity waves. Recently, O'Sullivan and Dunkerton (1995) emphasized the fact that baroclinic activity in the upper troposphere, which was intense when the rays crossed the tropopause, is likely to excite low-frequency gravity waves. The simulations made by O'Sullivan and Dunkerton (1995) showed that the favored locations for gravity wave emission are located on the western flank of the ridge, either in the "jet exit" region or west of the southernmost part of the high-PV tongue. The rays actually cross the tropopause in the vicinity of the trough. Moreover, the intrinsic frequency of the wave packet at the tropopause stays relatively low $(\leq 6 f$, the mean for all the waves which reached the tropopause is $5.5 \mathrm{f}$ ), therefore, supporting the emission by geostrophic adjustment near the $340 \mathrm{~K}$ isentrope. Note also that Pavelin et al. (2001) argued that geostrophic adjustment was responsible for the generation of the inertia-gravity waves that they observed in the lowermost stratosphere in a very similar synoptic situation (their Fig. 4).

We are now examining whether the ECMWF analyses support the likelihood of geostrophic adjustment close to the region of presumed generation. A measure of the departure from the geostrophic balance may be obtained through the calculation of the Lagrangian Rossby number, which is defined by (e.g. Koch and Dorian, 1988; O'Sullivan and Dunkerton, 1995):

$R o=\frac{\left|\frac{d \boldsymbol{v}_{h}}{d t}\right|}{f\left|\boldsymbol{v}_{h}\right|}$

where $\boldsymbol{v}_{h}$ is the horizontal wind vector. The map of the Lagrangian Rossby number on the $340 \mathrm{~K}$ isentropic surface at 


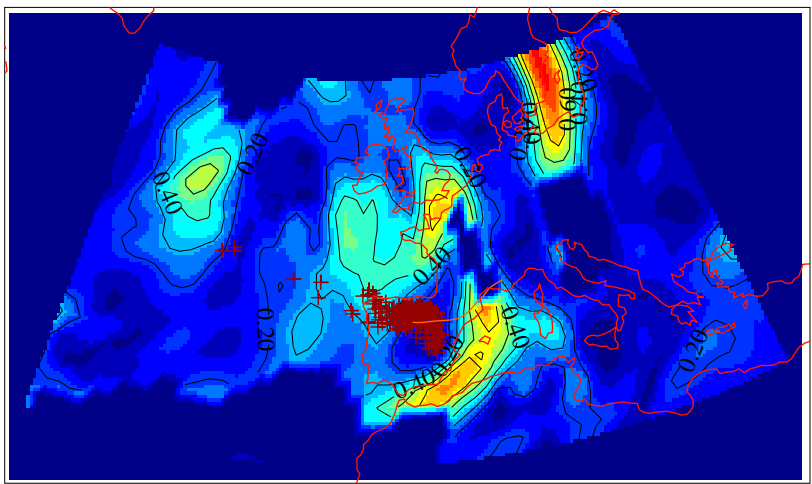

Fig. 9. Lagrangian Rossby number on the $340 \mathrm{~K}$ isentrope on 2 September 1997 at 14:00 UTC. Isocontours are drawn for $R o=$ $0.1,0.2, \ldots$ The Rossby number was not calculated when $\boldsymbol{v}_{h} \leq$ $10 \mathrm{~m} \mathrm{~s}^{-1}$. The trajectory of the "observed ray" is also shown (orange curve), as well as the advected intersection of the rays with that surface (crosses).

the supposed time of generation is presented in Fig. 9 (note that Ro was not computed when $\boldsymbol{v}_{h} \leq 10 \mathrm{~m} \mathrm{~s}^{-1}$ to avoid spurious large values). Though the simulated rays did not reach the regions of maximum imbalance, they did cross that isentrope which was close to them. In addition, O'Sullivan and Dunkerton (1995) found that gravity wave generation also occurred in regions where the Lagrangian Rossby number was $\sim .2$

Yet Zhang et al. (2000) argued that the Lagrangian Rossby number is only a basic imbalance indicator, and that for highly-curvated jets (as is the case here), it is more relevant to look at the residual of the nonlinear balance equation (NBE). In pressure coordinates, the NBE is:

$$
2 J(u, v)-\beta u+f \zeta-\nabla^{2} \Phi=0
$$

where $J$ stands for the Jacobian, $u$ and $v$ are, respectively, the zonal and meridional components of the horizontal wind, $\zeta$ is the relative vorticity, and $\Phi$ is the geopotential. The residual of the NBE is shown in Fig. 10 on the $190 \mathrm{hPa}$ isobaric surface, which is located close to the $340 \mathrm{~K}$ isentrope. The region of maximum imbalance is located somewhat eastward of the region where the wave packets were presumably generated. Note also that the maximum imbalance does not exactly correspond to the maximum Lagrangian Rossby number.

Another approach is to look directly at the divergence of the horizontal wind in the ECMWF model. This approach has been used by (O'Sullivan and Dunkerton, 1995) to support gravity waves in their model. Indeed, the gravity wave that we are looking at has horizontal and vertical wavelengths $(\approx 600 \mathrm{~km}$ and $\approx 10 \mathrm{~km}$, respectively) larger than the ECMWF model resolution. Furthermore, the model time step is of the order of some minutes, i.e. much shorter than the wave period. Therefore, the model is intrinsically able to capture the wave. However, the horizontal-velocity divergence in the GCMs are generally filtered in order to avoid a spurious energy cascade toward the smallest scales. This
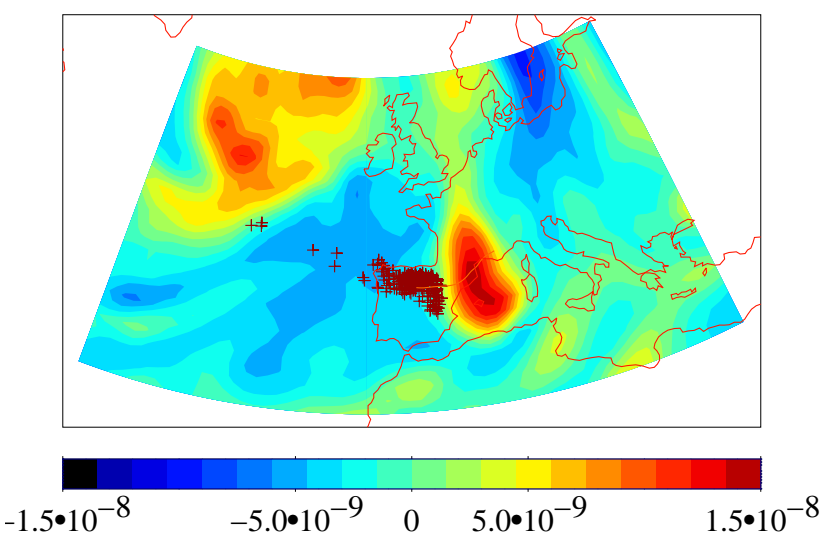

$\left(\mathrm{s}^{-2}\right)$

Fig. 10. Residual of the nonlinear balance equation at $190 \mathrm{hPa}$, on 2 September 1997 at 14:00 UTC. The intersection of the rays with the $190 \mathrm{hPa}$ surface and the observed ray are shown as in Fig. 9.
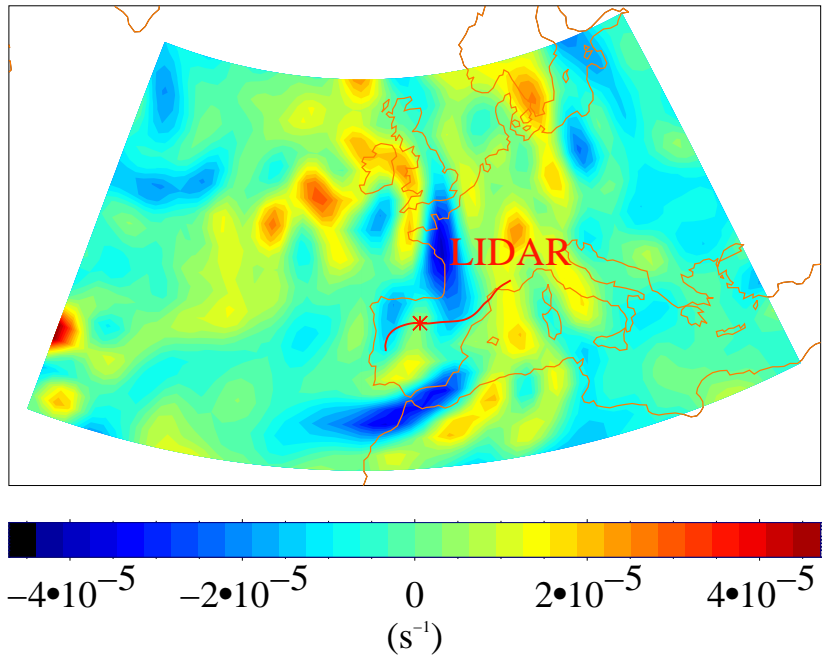

Fig. 11. Divergence of the horizontal-velocity analyzed field on 2 September 1997 at 14:00 UTC. The trajectory of the observed ray is also shown.

may actually dampen gravity waves in general circulation models (and incidentally also modify the structure of the generation indices that we computed above). Nevertheless, close to its generation region, one can expect some signature of the inertio-gravity wave in the simulated fields. Therefore, we examined the horizontal velocity divergence at the assumed time of generation and on the $190 \mathrm{hPa}$ isobaric surface (Fig. 11).

The horizontal velocity divergence exhibits large fluctuations above western Europe, which are located close to the maximum-ageostrophism regions (compare with Figs. 9 and 10). This figure is also reminiscent of Fig. 3 of O'Sullivan and Dunkerton (1995). Indeed most of the wave patterns in Fig. 11 are located in the jet exit region or at the southernmost part of the trough. The observed ray crosses the isobaric surface in the southern part of the pattern associated with the jet 


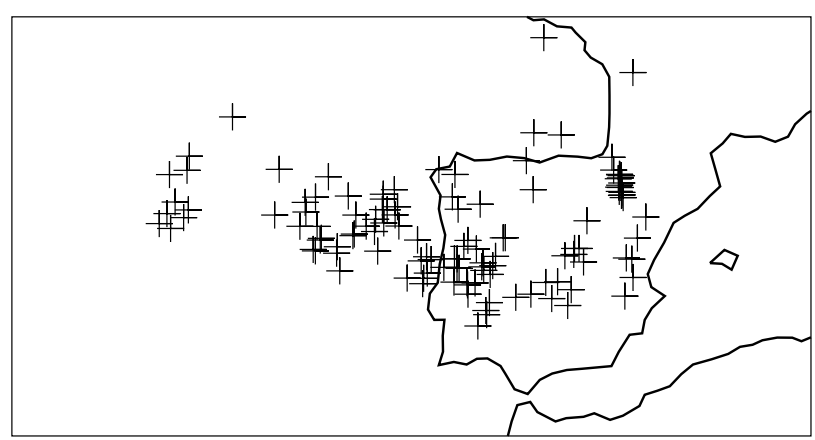

Fig. 12. Crosses indicate where the rays reached the ground surface.

exit region. The contours of horizontal velocity divergence in that region are primarily meridionally oriented. On the other hand, at that time, the meridional wavelength of the wave packet is twice as large as its zonal wavelength, which also implies that the phase contours are primarily meridionally oriented. Thus, the ray-tracing simulations are consistent with the characteristics of the inertio-gravity wave captured by the ECMWF model.

\subsection{Other processes}

As noted in the Introduction, one of the most studied gravitywave generation mechanisms is the interaction of a flow with orography. However, the evidence in our case suggest that this mechanism is not responsible for the wave observed by the lidar. First, the observed ray reaches the ground in the southwestern part of the Iberian peninsula, quite far away from significant orography, such as the Pyrénées or the Iberian system. Furthermore, the sensitivity study shows that most of the rays also reach the ground level far from orography (see Fig. 12).

Finally, the horizontal wind modulus at the ground is small ( $\sim 5 \mathrm{~m} \mathrm{~s}^{-1}$, see Fig. 4$)$ so that when combined with the little orography, the efficiency of orographic generation is expected to be weak.

Recent studies have emphasized the significance of gravity wave generation by convective systems (e.g. Alexander et al., 1995; Allen and Vincent, 1995). To understand the role of convection in the generation of the observed wave, we examined the infrared Meteosat images for the studied period, which are shown in Fig. 13. The convective activity developed far away from the various ray trajectories (see Fig. 7): the convective systems are located either north or east of the Iberian Peninsula. Thus, there seems to be no obvious connection between convective activity and the wave packet.

As the observed ray penetrates in the troposphere, it crossed two regions with weak Richardson $(R i)$ numbers, corresponding to the shears associated with the stratospheric and tropospheric flanks of the jet stream. The Richardson numbers never reached the critical value of 0.25 , which is necessary for instabilities to develop (Miles, 1961; Howard,
1961). However, the Richardson numbers might be overestimated due to the vertical resolution of the model at the tropopause $(\sim 500 \mathrm{~m})$. Moreover, even for $R i \sim 1$, Lott (1997) shows that small perturbations of a stable stratified shear layer may present significant grown rates and generate propagating gravity waves. Therefore, the hypothesis that the gravity wave observed by the lidar has been generated by shear instabilities cannot be ruled out.

\section{Summary and final remarks}

In this paper, we analyzed a Doppler lidar observation of a high-amplitude inertio-gravity wave in the mid-latitude lower stratosphere. Ray-tracing calculations with realistic atmospheric conditions have been performed to localize the region of the flow where the wave may have been generated. It appeared that the upper-tropospheric circulation was disturbed by baroclinic activity in the region where the rays crossed the tropopause. Some areas of significant departure from geostrophic equilibrium have been identified. These results tend to support that the wave could have been generated during the geostrophic adjustment of the background flow. Furthermore, the generation location (on the western flank of the trough, near the jet exit region) and the uppertropospheric synoptic circulation are very close to the simulations realized by O'Sullivan and Dunkerton (1995), which actually captured the generation of inertia-gravity waves during the decay phase of baroclinic instabilities. However, it is also possible that the wave generation was linked to the large shears associated with the tropospheric jet stream.

Nastrom et al. (1997) and Hertzog et al. (2001) have shown that gravity wave activity in the mid-latitude lower stratosphere is highly variable on time scales that are comparable with typical time scales of the synoptic tropospheric circulation (some days to some weeks). Baroclinic activity in the upper troposphere, to which we attributed the observed inertio-gravity wave generation, may thus be responsible for part of this gravity wave variability. Furthermore, it should be noted that the wave that we observed propagated a significant horizontal distance from its generation location, and that during that time, the upper-tropospheric trough had decayed. The tropospheric jet stream was thus relatively weak in the lidar observations. This may support the fact that Nastrom et al. (1997) did not find any significant correlation between the wave activity in the lower stratosphere and the simultaneous intensity of the tropospheric jet.

Finally, western Europe is located at the end of the north Atlantic storm track, so that it is certainly a favored location for observing such inertia-gravity waves. For instance, Thomas et al. (1999) and Pavelin et al. (2001) reported on radar observations of inertia-gravity waves in circumstances that are similar to those presented here: baroclinic activity in the upper troposphere, and regions of departure from geostrophic equilibrium. 
01/09/1997 18 UTC

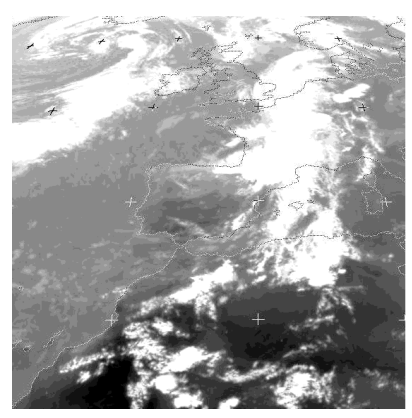

02/09/1997 18 UTC

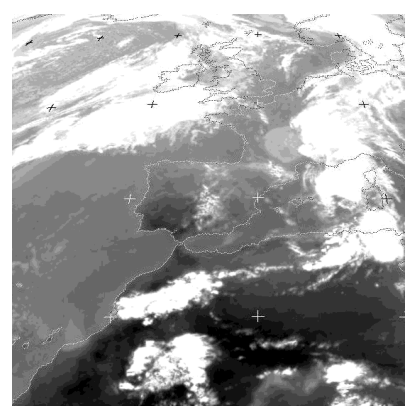

02/09/1997 6 UTC

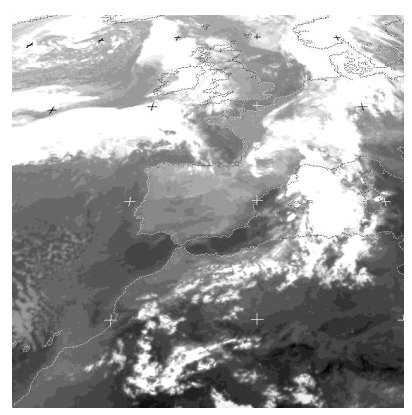

03/09/1997 6 UTC
02/09/1997 12 UTC

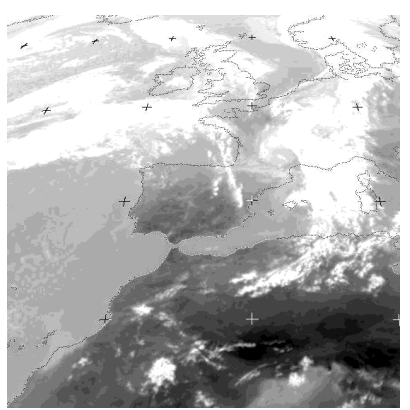

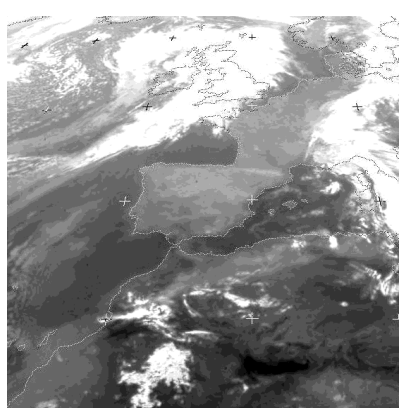

Fig. 13. Meteosat infrared images of western Europe.

\section{Appendix A}

Equations of ray-tracing

For clarity, system (5) which describes the trajectory of the wave packet and the variation of its wavenumbers along the ray is recalled:

$\frac{d x_{i}}{d t}=\frac{\partial \omega}{\partial k_{i}} \quad(i=1,2,3)$

$\frac{d k_{i}}{d t}=-\frac{\partial \omega}{\partial x_{i}}$

The gravity wave dispersion relation is:

$m^{2}=\frac{N^{2}-\omega_{0}^{2}}{\omega_{0}^{2}-f^{2}}\left(k^{2}+l^{2}\right)$

and it is only valid for a vertical wavelength smaller than, say, $25 \mathrm{~km}$. Finally, the apparent frequency is given by:

$\omega=\omega_{0}+k \bar{u}+l \bar{v}$

Since we are concerned with the trajectory of a gravity wave in the real atmosphere, we must take into account the threedimensional structure of the background fields, as well as their non-stationarity. Let $k_{t}=\sqrt{k^{2}+l^{2}+m^{2}}, k_{h}=$ $\sqrt{k^{2}+l^{2}}$ and $R=\frac{k_{h}^{2}}{2 k_{t}^{2}}$. System (A1) may be written as:

$$
\begin{aligned}
& \frac{d x}{d t}=\frac{k m^{2}\left(N^{2}-f^{2}\right)}{\omega_{0} k_{t}^{4}}+\bar{u} \\
& \frac{d y}{d t}=\frac{l m^{2}\left(N^{2}-f^{2}\right)}{\omega_{0} k_{t}^{4}}+\bar{v}
\end{aligned}
$$

$$
\begin{gathered}
\frac{d z}{d t}=\frac{-m k_{h}^{2}\left(N^{2}-f^{2}\right)}{\omega_{0} k_{t}^{4}} \\
\frac{d k}{d t}=-\frac{R N_{x}^{2}}{\omega_{0}}+k \bar{u}_{x}+l \bar{v}_{x} \\
\frac{d l}{d t}=-\frac{R N_{y}^{2}}{\omega_{0}}+k \bar{u}_{y}+l \bar{v}_{y} \\
\frac{d m}{d t}=-\frac{R N_{z}^{2}}{\omega_{0}}+k \bar{u}_{z}+l \bar{v}_{z}
\end{gathered}
$$

where subscripts stand for derivation. The evolution of the apparent frequency follows:

$\frac{d \omega}{d t}=\frac{k_{h}^{2} N_{t}^{2}}{\omega_{0} k_{t}^{2}}+k \bar{u}_{t}+l \bar{v}_{t}$.

With this set of equations, the variations in the wave vector are only known along the ray path, whereas the estimation of the WKB parameters $(\alpha, \beta, \gamma, \delta$ defined in Eq. 7) demands the calculation of local derivatives of the wave vector.

In addition, the calculation of the action density evolution also requires that one is able to estimate local derivatives (Hayes, 1970) of the trajectory; this is shown below. Let the trajectory of the wave packet be written with upper case letters $(X, Y, Z)$, and the space coordinates with lower case: $(x, y, z)$. Hence, for instance, $X=X(x, y, z, t)$. Moreover, we write:

$\omega=\Omega(\boldsymbol{k}, \boldsymbol{x}, t)$

where $(\boldsymbol{k}, \boldsymbol{x}, t)$ is the so-called augmented space (Eckermann and Marks, 1996). 
The action density $(\mathcal{A})$ of the wave packet is related to its energy $(E)$ by:

$\mathcal{A}=E / \omega_{0}$

and follows a conservation law:

$$
\frac{d \mathcal{A}}{d t}+\mathcal{A} \nabla \cdot c_{g}=0
$$

so that it is related to the matrix $\nabla_{x} \boldsymbol{X}$, the matrix of the derivative of the trajectory versus the space coordinates:

$\mathcal{A}=\frac{K}{\left|\nabla_{x} \boldsymbol{X}\right|}$

where $K$ is a constant, which is fixed, for instance, by initial conditions, and $\left|\nabla_{x} \boldsymbol{X}\right|$ is the Jacobian determinant of $\nabla_{x} \boldsymbol{X}$. The advantage of computing the action density from the Jacobian is that when the ray path encounters a caustic (i.e. when the volume occupied by the wave packet in the physical space vanishes), the ray theory fails and predicts infinite values for the action density, while the Jacobian remains finite.

Therefore, it is convenient to derive equations for computing the evolution of $\nabla_{x} \boldsymbol{X}$ and $\nabla_{x} \boldsymbol{k}$. Those equations are:

$$
\begin{aligned}
& \frac{d \nabla_{x} \boldsymbol{X}}{d t}=\nabla_{x} \boldsymbol{X} \cdot \Omega_{x k}+\nabla_{x} \boldsymbol{k} \cdot \Omega_{k k} \\
& \frac{d \nabla_{x} \boldsymbol{k}}{d t}=-\nabla_{x} \boldsymbol{X} \cdot \Omega_{x x}-\nabla_{x} \boldsymbol{k} \cdot \Omega_{k x}
\end{aligned}
$$

The matrix $\Omega_{x x}, \Omega_{x k}$ and $\Omega_{k k}$ are described below and we have $\Omega_{k x}={ }^{t} \Omega_{x k}$. It follows that:

$$
\Omega_{x_{i} x_{j}}=\frac{R}{\omega_{0}^{3}}\left(N_{x_{i} x_{j}}^{2} \omega_{0}^{2}-R N_{x_{i}}^{2} N_{x_{j}}^{2}\right)+k \bar{u}_{x_{i} x_{j}}+l \bar{v}_{x_{i} x_{y}}(\mathrm{~A}
$$

and

$$
\begin{gathered}
\Omega_{k x_{i}}=\frac{k m^{2} N_{x_{i}}^{2}}{\omega_{0}^{3} k_{t}^{4}}\left(\omega_{0}^{2}-R\left(N^{2}-f^{2}\right)\right)+\bar{u}_{x_{i}} \\
\Omega_{l x_{i}}=\frac{l m^{2} N_{x_{i}}^{2}}{\omega_{0}^{3} k_{t}^{4}}\left(\omega_{0}^{2}-R\left(N^{2}-f^{2}\right)\right)+\bar{v}_{x_{i}} \\
\Omega_{m x_{i}}=\frac{-m k_{h}^{2} N_{x_{i}}^{2}}{\omega_{0}^{3} k_{t}^{4}}\left(\omega_{0}^{2}-R\left(N^{2}-f^{2}\right)\right) .
\end{gathered}
$$

Finally, $\Omega_{k k}$ is symmetric, so that we only specify its six upper elements:

$$
\begin{array}{r}
\Omega_{k k}=\frac{m^{2}\left(N^{2}-f^{2}\right)}{\omega_{0}^{3} k_{t}^{8}}\left[\omega_{0}^{2} k_{t}^{4}-k^{2} m^{2}\left(N^{2}-f^{2}\right)-\right. \\
\left.4 k^{2} \omega_{0}^{2} k_{t}^{2}\right] \\
\Omega_{k l}=\frac{-k l m^{2}\left(N^{2}-f^{2}\right)}{\omega_{0}^{3} k_{t}^{8}}\left[m^{2}\left(N^{2}-f^{2}\right)+4 \omega_{0}^{2} k_{t}^{2}\right] \\
\Omega_{k m}=\frac{k m\left(N^{2}-f^{2}\right)}{\omega_{0}^{3} k_{t}^{8}}\left[2 \omega_{0}^{2} k_{t}^{4}-m^{2} k_{h}^{2}\left(f^{2}-N^{2}\right)-\right. \\
\left.4 m^{2} \omega_{0}^{2} k_{t}^{2}\right]
\end{array}
$$

$$
\begin{array}{r}
\Omega_{l l}=\frac{m^{2}\left(N^{2}-f^{2}\right)}{\omega_{0}^{3} k_{t}^{8}}\left[\omega_{0}^{2} k_{t}^{4}-l^{2} m^{2}\left(N^{2}-f^{2}\right)-\right. \\
\left.4 l^{2} \omega_{0}^{2} k_{t}^{2}\right]
\end{array}
$$

$$
\begin{array}{r}
\Omega_{l m}=\frac{\operatorname{lm}\left(N^{2}-f^{2}\right)}{\omega_{0}^{3} k_{t}^{8}}\left[2 \omega_{0}^{2} k_{t}^{4}-m^{2} k_{h}^{2}\left(f^{2}-N^{2}\right)-\right. \\
\left.4 m^{2} \omega_{0}^{2} k_{t}^{2}\right]
\end{array}
$$

$$
\begin{array}{r}
\Omega_{m m}=\frac{k_{h}^{2}\left(f^{2}-N^{2}\right)}{\omega_{0}^{3} k_{t}^{8}}\left[\omega_{0}^{2} k_{t}^{4}-m^{2} k_{h}^{2}\left(f^{2}-N^{2}\right)-\right. \\
\left.4 m^{2} \omega_{0}^{2} k_{t}^{2}\right] .
\end{array}
$$

Initially, the matrices are set as:

$$
\begin{aligned}
\nabla_{x} \boldsymbol{X} & =\mathcal{I} \\
\nabla_{x} \boldsymbol{k} & =0
\end{aligned}
$$

where $\mathcal{I}$ is the identity matrix, which corresponds to plane waves. By doing this we assume that at the initial altitude, the wind and static stability fields field can be considered as quasi-homogeneous. In the lower stratosphere, it is expected that the vertical variation of horizontal wind induces the main departure from homogeneity. However, Fig. 1 shows that at $19 \mathrm{~km}$, the vertical shear is relatively weak, so that our assumption may be valid.

Acknowledgement. The authors acknowledge the ECMWF for providing the T106 model results. The authors are also very grateful to the technical staff at Verrières and at the OHP for the maintenance and the operation of the Doppler lidar. Discussions with H. Teitelbaum and F. Vial at LMD, as well as the comments of one anonymous reviewer, have greatly contributed to the improvement of this paper.

Topical Editor J.-P. Duvel thanks S. Koch for his help in evaluating this paper.

\section{References}

Alexander, M. J., Holton, J. R., and Durran, D. R., The gravity wave response above deep convection in a squall line simulation, J. Atmos. Sci., 52, 2212-2226, 1995.

Alexander, M. J. and Rosenlof, K. H., Nonstationary gravity wave forcing of the stratospheric zonal mean wind, J. Geophys. Res., 101, 23 465-23 474, 1996.

Allen, S. J. and Vincent, R. A., Gravity wave activity in the lower atmosphere: Seasonal and latitudinal variations, J. Geophys. Res., 100, 1327-1350, 1995.

Andersson, E., Haseler, J., Undén, P., Courtier, P., Kelly, G., Vasiljević, D., Branković, C., Cardinali, C., Gaffard, C., Hollingsworth, A., Jakob, C., Janssen, P., Klinker, E., Lanzinger, A., Miller, M., Rabier, F., Simmons, A., Strauss, B., Théphault, J.-N., and Viterbo, P., The ECMWF implementation of threedimensional variational assimilation (3D-Var). III: Experimental results, Q. J. R. Meteorol. Soc., 124, 1831-1860, 1998.

Andrews, D. G., Holton, J. R., and Leovy, C. B., Middle Atmosphere Dynamics, Academic, San Diego, Calif., 1987.

Blumen, W., Geostrophic adjustment, Rev. Geophys. Space Phys., 10, 485-528, 1972. 
Bowman, K. P., Rossby waves phase speeds and mixing barriers in the stratosphere. Part I: Observations, J. Atmos. Sci., 53, 905916, 1996.

Broutman, D., On internal wave caustics, J. Phys. Oceanogr., 16, 1625-1635, 1986.

Cadet, D. and Teitelbaum, H., Observational evidence of internal inertial-gravity waves in the tropical stratosphere, J. Atmos. Sci., 36, 892-907, 1979.

Danielsen, E. F., Hipskind, R. S., Starr, W. L., Vedder, J. F., Gaines, S. E., Kley, D., and Kelly, K. K., Irreversible transport in the stratosphere by internal waves of short vertical wavelength, J. Geophys. Res., 96, 17433-17 452, 1991.

Eckermann, S. D., Gison-Wilde, D. E., and Bacmeister, J. T., Gravity wave perturbations of minor constituents: A parcel advection methodology, J. Atmos. Sci., 55, 3521-3539, 1998.

Eckermann, S. D. and Marks, C. J., An idealized ray model of gravity wave-tidal interactions, J. Geophys. Res., 101, 21 195$21212,1996$.

Eckermann, S. D. and Vincent, R. A., VHF radar observations of gravity-waves production by cold fronts over southern Australia, J. Atmos. Sci., 50, 785-806, 1993.

Ford, R., McIntyre, M. E., and Norton, W. A., Balance and the slow quasimanifold: some explicit results, J. Atmos. Sci., 57, 12361254, 2000.

Fritts, D. C., Shear excitation of atmospheric gravity waves, J. Atmos. Sci., 39, 1936-1952, 1982.

Fritts, D. C. and Luo, Z., Gravity waves excitation by geostrophic adjustment of the jet stream. Part I: Two-dimensional forcing, J. Atmos. Sci., 49, 681-697, 1992.

Fritts, D. C. and Nastrom, G. D., Sources of mesoscale variability of gravity waves. Part II: frontal, convective and jet stream excitation, J. Atmos. Sci., 49, 111-127, 1992.

Gall, R. L., Williams, R. T., and Clark, T. L., Gravity waves generated during frontogenesis, J. Atmos. Sci., 45, 2204-2219, 1988.

Gibson-Wilde, D. E., Vincent, R. A., Souprayen, C., Godin, S., Hertzog, A., and Eckermann, S. D., Dual lidar observations of mesoscale fluctuations of ozone and horizontal winds, Geophys. Res. Lett., 24, 1627-1630, 1997.

Griffiths, M. and Reeder, M. J., Stratospheric inertia-gravity waves generated in a numerical model of frontogenesis. I: Model solutions, Q. J. R. Meteorol. Soc., 122, 1153-1174, 1996.

Hayes, W. D., Kinematic wave theory, Proc. Roy. Soc. Lond., A320, 209-226, 1970.

Hertzog, A., Souprayen, C., and Hauchecorne, A., Measurements of gravity wave spectra in the lower stratosphere by Doppler lidar, J. Geophys. Res., 106, 7879-7890, 2001.

Holton, J. R., Haynes, P. H., McIntyre, M. E., Douglass, A. R., Hood, R. B., and Pfister, L., Stratosphere-troposphere exchange, Rev. Geophys., 33, 405-439, 1995.

Howard, L. N., Note on a paper of J. W. Miles, J. Fluid Mech., 10, 509-512, 1961.

Jones, W. L., Ray Tracing for internal gravity waves, J. Geophys. Res., 8, 2028-2033, 1969.

Koch, S. E. and Dorian, P. B., A mesoscale gravity wave event observed during CCOPE. Part III: Wave environment and probable source mechanisms, Mon. Wea. Rev., 116, 2570-2592, 1988.

Lait, L. R., An alternative form for potential vorticity, J. Atmos. Sci., 51, 1754-1759, 1994.

Lalas, D. P. and Einaudi, F., On the characteristics of gravity waves generated by atmospheric shear layers, J. Atmos. Sci., 33, 12481259, 1976.

Ley, B. E. and Peltier, W. R., Wave generation and frontal collapse,
J. Atmos. Sci., 35, 3-17, 1978.

Lighthill, M. J., Waves in Fluids, Cambridge University Press, 1978, 504pp.

Lott, F., The transient emission of prapagating gravity waves by a stably stratified shear layer, Q. J. R. Meteorol. Soc., 123, 16031619, 1997.

Lott, F. and Teitelbaum, H., Topographic waves generated by a transient wind, J. Atmos. Sci., 50, 2607-2624, 1993.

Lu, D., VanZandt, T. E., and Clark, W. L., VHF Doppler radar observations of buoyancy waves associated with thunderstorms, J. Atmos. Sci., 41, 272-282, 1984.

Luo, Z. and Fritts, D. C., Gravity waves excitation by geostrophic adjustment of the jet stream. Part II: Three-dimensional forcing, J. Atmos. Sci., 50, 104-115, 1993.

Marks, C. J. and Eckermann, S. D., A three-dimensional nonhydrostatic ray-tracing model for gravity waves: Formulation and preliminary results for the middle atmosphere, J. Atmos. Sci., 52, 1959-1984, 1995.

Miles, J. W., On the stability of heterogeneous shear flows, J. Fluid Mech., 10, 496-508, 1961.

Nastrom, G. D. and Fritts, D. C., Sources of mesoscale variability of gravity waves, Part I, Topographic excitation, J. Atmos. Sci., 49, 101-110, 1992.

Nastrom, G. D., VanZandt, T. E., and Warnock, J. M., Vertical wavenumber spectra of wind and temperature from highresolution balloon soundings over Illinois, J. Geophys. Res., 102, 6685-6701, 1997.

O'Sullivan, D. and Dunkerton, T. J., Generation of inertia-gravity waves in a simulated life cycle of baroclinic instability, J. Atmos. Sci., 52, 3695-3716, 1995.

Pavelin, E., Whiteway, J. A., and Vaughan, G., Observation of gravity wave generation and breaking in the lowermost stratosphere, J. Geophys. Res., 106, 5173-5179, 2001.

Pfister, L., Starr, W., Craig, R., Loewenstein, M., and Legg, M., Small-scale motions observed by aircraft in the tropical lower stratosphere: evidence for mixing and its relationship to largescale flows, J. Atmos. Sci., 43, 3210-3225, 1986.

Press, W. H., Flannery, B. P., Teukolvsky, S. A., and Vetterling, W. T., Numerical recipes: The art of scientific computing, Cambridge University Press, 1989, 702pp.

Reeder, M. J. and Griffiths, M., Stratospheric inertia-gravity waves generated in a numerical model of frontogenesis, II, Wave sources, generation mechanisms and momentum fluxes, Q. J. R. Meteorol. Soc., 122, 1175-1195, 1996.

Sato, K., An inertial gravity wave associated with a synoptic-scale pressure trough observed by the MU Radar, J. Meteorol. Soc. Jpn., 67, 325-333, 1989.

Sato, K., A statistical study of the structure, saturation and sources of inertio-gravity waves in the lower stratosphere observed with the MU radar, J. Atmos. Terr. Phys., 56, 755-774, 1994.

Sato, T. and Woodman, R. F., Fine altitude resolution radar observations of upper-tropospheric and lower-stratospheric winds and waves, J. Atmos. Sci., 39, 2539-2545, 1982.

Souprayen, C., Garnier, A., Hertzog, A., Hauchecorne, A., and Porteneuve, J., Doppler wind lidar for stratospheric measurements, Part I, Instrumental setup-validation-first climatological results, Appl. Opt., 38, 2410-2421, 1999.

Sutherland, B. R. and Peltier, W. R., Internal gravity wave emission into the middle atmosphere from a model tropospheric jet, J. Atmos. Sci., 52, 3214-3235, 1995.

Teitelbaum, H., Moustaoui, M., Ovarlez, J., and Kelder, H., The role of atmospheric waves in the laminated structure of ozone 
profiles at high latitudes, Tellus, 48A, 442-455, 1996.

Teitelbaum, H., Ovarlez, J., Kelder, H., and Lott, F., Some observations of gravity-wave induced structure in ozone and water vapour during EASOE, Geophys. Res. Lett., 21, 1483-1486, 1994.

Thomas, L., Prichard, I. T., and Austin, I., Radar observations of an inertia-gravity wave in the troposphere and lower stratosphere, Ann. Geophys., 10, 690-697, 1992.

Thomas, L., Worthington, R. M., and McDonald, A. J., Inertiagravity waces in the troposphere and lower stratosphere assiocated with a jet stream exit region, Ann. Geophys., 17, 115-121, 1999.

Thompson, R. O. R. Y., Observation of inertial waves in the stratosphere, Q. J. R. Meteorol. Soc., 104, 691-698, 1978.
Thorncroft, C. D., Hoskins, B. J., and McIntyre, M. E., Two paradigms of baroclinic-wave life-cycle behaviour, Q. J. R. Meteorol. Soc., 119, 17-55, 1993.

Van Tuyl, A. H. and Young, J. A., Numerical simulation of nonlinear jet streak adjustment, Mon. Wea. Rev., 110, 2038-2054, 1982.

Waugh, D. W., Plumb, R. A., Atkinson, R. J., Schoeberl, M. R., Lait, L. R., Newman, P. A., Loewenstein, M., Toohey, D. W., Avallone, L. M., Webster, C. R., and May, R. D., Transport out of the lower stratospheric Arctic vortex by Rossby wave breaking, J. Geophys. Res., 99, 1071-1088, 1994.

Zhang, F., Koch, S. E., Davies, C. A., and Kaplan, M. L., A survey of unbalanced flow diagnostics and their application, Adv. Atmos. Sci., 17, 165-183, 2000. 